Research Paper

\title{
A Formulation Study of 5-Aminolevulinic Encapsulated in DPPC Liposomes in Melanoma Treatment
}

\author{
Ming-Wei Lin ${ }^{1}$, Yaw-Bin Huang1, 2, Chun-Lin Chen ${ }^{1,3}$, Pao-Chu $\mathrm{Wu}^{2}$,Chien-Ying Chou ${ }^{2}$, Ping-Ching \\ $\mathrm{Wu}^{4,5,6}$, Shih-Ya Hung7,8, \\ 1. Center for Stem Cell Research, Kaohsiung Medical University, Kaohsiung 80756, Taiwan \\ 2. School of Pharmacy, Kaohsiung Medical University, Kaohsiung 80756, Taiwan \\ 3. Department of Biological Science, National Sun Yat-sen University, Kaohsiung 80424, Taiwan \\ 4. Department of Biomedical Engineering, National Cheng Kung University, Tainan, 701, Taiwan \\ 5. Institute of Oral Medicine and Department of Stomatology, National Cheng Kung University Hospital, College of Medicine, National Cheng Kung \\ University Tainan 701, Taiwan \\ 6. Medical Device Innovation Center, Taiwan Innovation Center of Medical Devices and Technology, National Cheng Kung University Hospital, National \\ Cheng Kung University, Tainan 701, Taiwan \\ 7. Graduate Institute of Integrated Medicine, College of Chinese Medicine, China Medical University, Taichung 40402, Taiwan \\ 8. Division of Colorectal Surgery, China Medical University Hospital, Taichung 40447, Taiwan
}

$\triangle$ Corresponding authors: Shih-Ya Hung, Graduate Institute of Integrated Medicine, College of Chinese Medicine, China Medical University, Taichung 40402, Taiwan. Tel: +886-4-22053366 ext. 3513. E-mail: shihyahung@mail.cmu.edu.tw, or Ping-Ching Wu, Department of Biomedical Engineering, National Cheng Kung University, Tainan, 701, Taiwan. Tel: +886-6-757575 ext. 63436. Fax: +886-6-2343270. E-mail: wbcxyz@gmail.com

(1) Ivyspring International Publisher. Reproduction is permitted for personal, noncommercial use, provided that the article is in whole, unmodified, and properly cited. See http://ivyspring.com/terms for terms and conditions.

Received: 2016.03.01; Accepted: 2016.05.08; Published: 2016.06.18

\begin{abstract}
Photodynamic therapy (PDT) is a widely used technique for epithelial skin cancer treatment. 5 -aminolevulinic acid (5-ALA) is a drug currently used for PDT and is a hydrophilic molecule at its physiological $\mathrm{pH}$, and this limits its capacity to cross the stratum corneum of skin. Since skin penetration is a key factor in the efficacy of topical 5-ALA-mediated PDT, numerous strategies have been proposed to improve skin penetration. Yet this problem is still ongoing. The results of a previous study showed a low rate of 5-ALA encapsulated in liposomes (5.7\%) that were $400 \mathrm{~nm}$ in size. In the present study, we used 1,2-dipalmitoyl-sn-glycero-3-phosphocholine (DPPC) liposomes as vehicles and tested their delivery efficacy of 5-ALA-medicated PDT both in vitro and in vivo. Our data shows that 5 -ALA encapsulated in 0.1 or $0.5 \%$ DPPC liposomes (5-ALA/DPPC) had a better encapsulated rate $(15 \sim 16 \%)$ and were smaller in size $(84 \sim 89 \mathrm{~nm})$. We found the 5-ALA/DPPC formulation reduced cell viability, mitochondria membrane potential, and enhanced intracellular ROS accumulation as compared to 5-ALA alone in melanoma cells. Furthermore, the 5-ALA/DPPC formulation also had better skin penetration ability as compared to the 5-ALA in our ex vivo data by assaying 5-ALA converted into protoporphyrin IX (PpIX) in the skin of the mice that were experimented on. In melanoma xenograft models, 5-ALA/DPPC enhanced PpIX accumulation only in tumor tissue but not normal skin. In conclusion, we found DPPC liposomes to be good carriers for 5-ALA delivery and believe that they may prove useful in 5-ALA-mediated PDT in the future.
\end{abstract}

Key words: 5-aminolevulinic acid, DPPC liposomes, melanoma, photodynamic therapy, skin cancer

\section{Introduction}

Skin cancer is the most common human cancer with over a million cases detected every year [1]. Risk factors for skin cancer include ultraviolet (UV) radiation from sun exposure, receiving an organ transplant, HIV, genetic factors, etc. [2]. Melanoma is the deadliest form of skin cancer. It is an aggressive, therapy-resistant malignancy of melanocytes and inclined to metastasize quickly into the lymphatic system and other organs such as the lungs, the liver, the brain, or the bones $[3,4]$. Incidences of melanoma have been steadily increasing worldwide, resulting in an increasing public health problem; and current 
chemotherapy; and chemo-immunotherapy regimes have shown little clinical benefit with no improvement in overall survival [4].

Photodynamic therapy (PDT) is a popular and non-invasive treatment based on selectively accumulating a non-toxic photosensitizing drug (e.g., 5-aminolevulinic acid and methyl aminolevulinate), a drug which will preferentially accumulate in tumorous tissue rather than normal tissue [5]. Subsequent activation by $635 \mathrm{~nm}$ light irradiation converts 5-ALA into protoporphyrin IX (PpIX) and initiates a photodynamic reaction via generating highly reactive singlet oxygen $\left({ }^{1} \mathrm{O}_{2}\right)$, and this process results in tumor cell death [6]. As a novel therapy, PDT has the advantages of being able to select for tumor cells and not destroy normal cells, and of not being limited by the location or size of skin lesions [7]. PDT has proven to have several benefits including low morbidity, minimal functional disturbance, better cosmetic outcomes, and the ability to repeatedly be used many times in one instance [8,9]. PDT is a successful treatment for non-melanoma skin cancers in clinical practice.[7] More and more doctors are using PDT to cure patients with skin cancer, especially elderly patients [7].

There are now increasing numbers of photosensitizing prodrugs being used clinically; and 5-aminolevulinic acid (5-ALA)-mediated PDT is one of the fastest developing areas in PDT [6]. 5-ALA is a naturally occurring delta amino acid that is ultimately converted in to PpIX, the immediate precursor of heme [5]. Topical 5-ALA PDT has shown significant efficacy in superficial skin cancer, actinic keratosis, psoriasis, cutaneous T-cells lymphoma, basal cell carcinoma, and squamous cell carcinoma [10-13]. However, 5-ALA is a $167.6 \mathrm{~g} / \mathrm{mol}$ hydrophilic molecule with a low penetration rate through intact skin.[14] Thus, recent investigations have focused on the development of carriers of 5-ALA that are able to penetrate intact skin. Enhancement of 5-ALA skin penetration via the improvement of the transdermal drug delivery system is a key factor in the success of topical applied PDT [15]. Enhancing techniques to increase transdermal drug delivery include chemical modification of 5-ALA, pretreatment with physical or chemical penetration enhancers (e.g., lasers, ultrasound, DMSO, EDTA), and penetration using different vehicles (e.g., lotion, liposomes) [15]. Of the aforementioned, liposomes are one of the best delivery systems for low-molecular-weight drugs, DNA, siRNA, etc.[16]. Liposomes are membrane-like phospholipid bilayers with a water phase inside that can carry both lipophilic and hydrophilic agents and are generally accepted in various delivery strategies for the systemic or topical administration of drugs
[17]. However, low 5-ALA encapsulation in liposomes has been reported, with Pierre et al demonstrating that 5-ALA-entrapped in stratum corneum lipid liposomes (SCLLs) formulation was only 5.7\% [18].

Since PDT is now a well-established treatment modality for cutaneous carcinomas and is based on the administration of a light-activated drug followed by the illumination of the pathological area, the treatment of metastatic melanoma remains a therapeutic challenge [19]. The aim of this study is to evaluate the in vitro and in vivo efficacy of the 1,2-dipalmitoyl-sn-glycero-3-phosphocholine (DPPC) liposomes formulation in the treatment of melanoma and gain more information about DPPC liposomes and their interaction with 5-ALA. The objective of this investigation was to create DPPC liposomes formulations as delivery systems that would be able to overcome the poor penetration of 5-ALA. WST-1 cell proliferation assay, ROS production and cellular mitochondrial membrane potential measurements were used to determine the photo-cytotoxicity of 5-ALA encapsulated in DPPC liposomes on melanoma cancer cells when exposed to PDT. The amount of 5-ALA skin penetration, as well as skin retention from DPPC liposomes, was investigated. Melanoma xenograft mouse models were employed to confirm liposomal-based PDT efficiency in vivo.

\section{Materials and Methods}

\section{Materials}

1,2-dipalmitoyl-sn-glycero-3-phosphocholine (DPPC) was obtained from Avanti (USA). Cholesterol was purchased from Sigma-Aldrich (USA). Other chemicals used in the study were reagent grade. Chemical structures of 5-ALA and DPPC are present in Figure 1A.

\section{Preparation of 5-ALA encapsulated in DPPC liposomes (5-ALA/DPPC)}

5-ALA encapsulated in DPPC liposomes (5-ALA/DPPC) was prepared according to the modified thin film hydration method [20]. DPPC and cholesterol were dissolved in chloroform and methanol $(3: 1, \mathrm{v} / \mathrm{v})$ in $250 \mathrm{~mL}$ round bottom flasks. The mixture was evaporated in a rotary evaporator above transition temperature, and solvent traces were removed under a vacuum overnight. The film was hydrated with $0.5 \%$ 5-ALA aqueous solution above lipid transition temperature for 30 minutes. The vesicle suspension was dispersed by a probe sonicator (UP50H, Germany) at 30\% amplitude for 15 minutes. For the diffusion experiments, DPPC liposomes loaded with $0.5 \%$ 5-ALA were prepared under the same conditions. 


\section{Characterization of 5-ALA encapsulated in DPPC liposomes}

The particle size and zeta potential of 5-ALA encapsulated in DPPC liposomes were measured by laser light-scattering with a helium-neon laser at 630 $\mathrm{nm}$ (Zetasizer $3000 \mathrm{HS}_{\mathrm{A}}$, Malvern, UK) at $25{ }^{\circ} \mathrm{C}$. The polydispersity index (PI) was used to measure size distribution. The entrapment efficiency of 5-ALA in DPPC liposomes was employed with an ultracentrifuge method (CS120GXL, Hitachi) at 60,000 $\mathrm{g}$ and $4{ }^{\circ} \mathrm{C}$ for 30 minutes. 5-ALA was analyzed by HPLC following centrifugation.

\section{Morphological determination of 5-ALA DPPC liposomes by transmission electron microscopy}

The 5-ALA/DPPC (0.5\% DPPC) was stained with a phosphotungstic acid (1\%) solution and titrated with a copper wire. After it had dried, its morphological structure was determined by transmission electron microscopy (TEM; Joel model JEO 2000 EX II, Japan)

\section{Cell culture}

Murine melanoma cells (B16F10) were obtained from the America Type Culture Collection (ATCC). B16F10 cells were allowed to grow to confluence using Dulbecco's modified Eagle's medium (DMEM) supplemented with $10 \%$ fetal bovine serum (FBS) and antibiotics $(100 \mathrm{U} / \mathrm{ml}$ of penicillin and $100 \mu \mathrm{g} / \mathrm{ml}$ of streptomycin).

\section{PDT and WST- 1 cell cytotoxicity assay}

Cell cytotoxicity of PDT was assessed using a WST-1 cell proliferation kit (Roche). Briefly, cells were seeded with $5 \times 10^{4}$ cells/well in a culture medium containing 5-ALA or 5-ALA/DPPC in 96-well microplates 4 hours before assay. 5-ALA in this culture medium was removed and replaced by PBS. An instrument with a light-emitting diode (LED) a red light source at $630 \mathrm{~nm}$ was used for PDT. A light dose of $50 \mathrm{~J} / \mathrm{cm}^{2}$ was delivered over $20 \mathrm{~min}$. Cell viability was measured $24 \mathrm{~h}$ later by WST-1 colorimetric assay with a microplate ELISA reader.

\section{Reactive oxygen species (ROS) measurement}

PDT-treated cells were then incubated with 1 $\mu \mathrm{M}$ superoxide-sensitive dye dihydroethidium. The ROS production during PDT was determined by FACScan flow cytometry (Becton Dickinson).

\section{Detection of mitochondrial transmembrane potential}

PDT-treated cells were incubated with $0.1 \mu \mathrm{M}$ Mitotraker (Invitrogen) for $30 \mathrm{~min}$ at $37^{\circ} \mathrm{C}$ in the dark.
The cells were washed with warm PBS, and Mitotraker fluorescence intensity was determined by FACScan flow cytometry (Becton Dickinson).

\section{Confocal laser scanning microscopy}

For confocal microscopy assay, the mice were treated with either the control (PBS), 5-ALA, 5 -ALA/DPPC $(0.5 \%$ DPPC). These were the three groups for our experiment. After 4 hours, the skin where the drug was administrated was harvested and fixed with $4 \%$ paraformaldehyde. Then tissue samples were sliced into $5 \mu \mathrm{m}$ sections by frozen section and mounted with Vectashield media (Vector Laboratories) for laser confocal microscopy observation (model FV1000, Olympus).

\section{Melanoma xenograft mouse models}

Six-week-old male BALB/c nu/nu nude mice were housed in a specific pathogen-free environment. The B16F10 cells were harvested, washed, and suspended in PBS. Then, $5 \times 10^{5}$ cells were injected subcutaneously into the right flank regions of the mice. The mice were then checked every day for the appearance of tumor, and the sizes of the tumors were determined by measuring 2 diameters with a caliper after PDT. Tumor volume (TV) was estimated using the equation TV $=(\text { length } x \text { width })^{2} / 2$ and expressed as a $\%$ of control. Three mice were included in each of the three groups.

\section{Results}

\section{Characteristics of 5-ALA encapsulated in DPPC liposomes (5-ALA/DPPC)}

After using the thin film method by addition of cholesterol as a stabilizer, the data of mean diameter (size), polydispersity index (representing the particle size), zeta potential, and entrapment efficiency of 5-ALA/DPPC were measured. As shown in Table 1, the size of 5 -ALA/0.1\% DPPC and 5-ALA/0.5\% DPPC were smaller than 5-ALA alone ( $0.5 \%$ final), with both being under $100 \mathrm{~nm}$. Moreover, polydispersity indexes were within 0.31 to 0.36 in the 5-ALA, 5-ALA/0.1\% DPPC and 5-ALA/0.5\% DPPC formulations. The 5-ALA/DPPC formulations achieved a cationic surface charge of 5-ALA that meant a tendency towards increasing contact with the cell membrane. The zeta potentials of the 5-ALA, 5-ALA/0.1\% DPPC, and 5-ALA/0.5\% DPPC liposomal formulations were about $3.40 \pm 0.80,6.60 \pm$ 0.50 and $15.10 \pm 0.70 \mathrm{mV}$, respectively. The encapsulation of 5-ALA $/ 0.1 \%$ DPPC and 5-ALA/0.5\% DPPC were similar (Table 1). This data indicates that DPPC enhances 5-ALA entrapment efficiency to $15-16 \%$, which is a substantial increase as 
compared to stratum corneum lipid liposomes (SCLLs, 5.7\%) [18].

Table 1. Physicochemical characteristics of 5-ALA-liposome

\begin{tabular}{lllll}
\hline Formulation & Size $(\mathrm{nm})$ & PI & Zeta $(\mathrm{mV})$ & EE $(\%)$ \\
\hline Blank & $114.20 \pm 1.70$ & $0.36 \pm 0.06$ & $3.40 \pm 0.80$ & - \\
$0.1 \%$ 5-ALA/DPPC & $84.50 \pm 8.08$ & $0.31 \pm 0.10$ & $6.60 \pm 0.50$ & $15.23 \pm 1.01$ \\
$0.5 \%$ 5-ALA/DPPC & $89.80 \pm 2.40$ & $0.36 \pm 0.01$ & $15.10 \pm 0.70$ & $16.43 \pm 2.53$ \\
\hline
\end{tabular}

PI: polydispersity index; EE: encapsulation efficiency

\section{In vitro analysis 5-ALA/DPPC produced photo-toxicity}

5-ALA administration with PDT produces PpIX accumulation in B16F10 melanoma cells in a dose-dependent manner [21]. Here, we studied whether DPPC as the 5-ALA carrier changes the PpIX accumulation in B16F10 cells. The photo-toxicity of 5-ALA-induced PpIX accumulation in B16F10 melanoma cells was determined by WST-1 to detect cell viability after PDT. As figure $2 \mathrm{~A}$ shows, the 5-ALA-treated cells' viability was reduced as compared to the control. 5-ALA/DPPC had lower cell viability than 5-ALA alone. The cell viability in 5-ALA and 5-ALA-DPPC were $52 \pm 12.8 \%$ and $33 \pm 9.4 \%$, respectively. It has been shown that ROS production after PDT leads to DNA fragmentation, mitochondria damage, and cell death [22]. Figures $2 \mathrm{~B} \& 2 \mathrm{C}$ show 5-ALA/DPPC-treated cells as having higher ROS production and lower mitochondrial membrane potential than 5-ALA-treated B16F10 cells. These data suggest that 5-ALA/DPPC has a higher photo-toxicity than 5-ALA in vitro.

\section{Morphology of 5-ALA/DPPC}

Since the physicochemical characteristics of 5-ALA/0.1\% DPPC and 5-ALA/0.5\% DPPC were similar, we further used TEM to study the morphology of 5-ALA/0.5\% DPPC. Figure 1B shows that 5-ALA/0.5\% DPPC had a relatively uniform size distribution which was consistent with the particle size data $(89.80 \pm 2.40 \mathrm{~nm})$ that we obtained using laser sizing.



Figure 1. The morphology of 5-ALA/DPPC by transmission electron microscopy (TEM). Molecular structures of 5-aminolevulinic acid (5-ALA) and 1,2-dipalmitoyl-sn-glycero-3-phosphocholine (DPPC). (B) The TEM image shows the morphology of 5-ALA encapsulated in DPPC (0.5\%, 5-ALA/DPPC) which was stained with $1 \%$ phosphotungstic acid before being analyzed by TEM. Scale bar $=500 \mathrm{~nm}$.
A

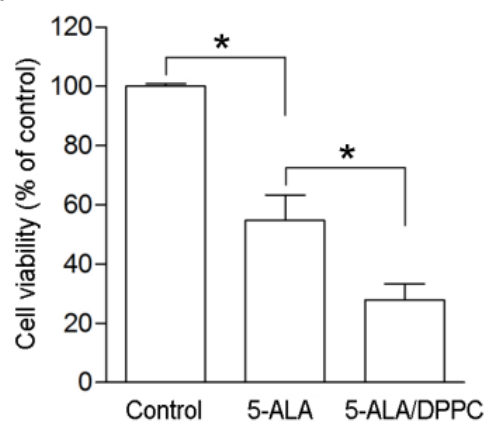

C

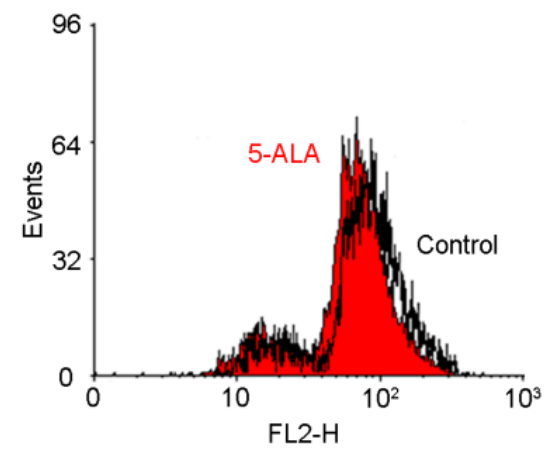

B
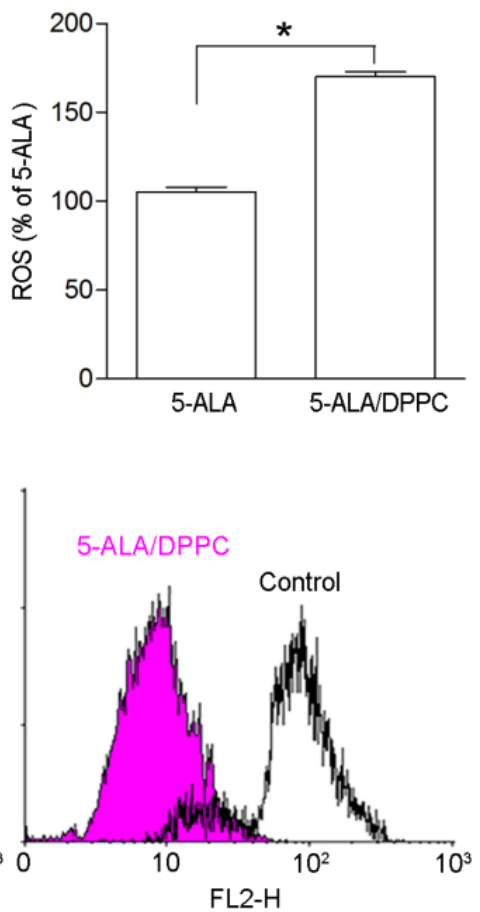

Figure 2. In vitro analysis 5-ALA/DPPC produced photo-toxicity. The production of reactive oxygen species after photodynamic therapy leads to DNA fragmentation, mitochondria damage, and cell death. Here, we used DPPC $(0.5 \%)$ as the 5-ALA carrier (5-ALA/DPPC) to study PpIX accumulation in B16F10 cells. (A) 5-ALA-treated B16F10 cells decreased in cell viability as compared to the control. And 5-ALA/DPPC-treated cells had lower cell viability as compared to 5-ALA-treated cells. The cell viability of 5-ALA and 5-ALA/DPPC groups were $52 \pm 12.8 \%$ and $33 \pm 9.4 \%$, respectively. (B) \& (C) show 5-ALA/DPPC-treated cells had a higher ROS production and lower mitochondrial membrane potential than 5-ALA-treated B16F10 cells. *, p $<0.05$. 



Figure 3. 5-ALA/DPPC-induced PpIX accumulation in ex vivo mice skin model. Since the accumulation of PplX emits a red fluorescence, we performed a confocal microscopy to assay the 5-ALA-induced PpIX accumulation after PDT in the skin of the mouse. After 4 hours of incubation, the PpIX intensity (red fluorescence) from 5-ALA/DPPC (0.5\% DPPC)-treated skin was stronger than that in 5-ALA. Scale bar $=100 \mu \mathrm{m}$. Here we used DAPI (blue) to stain the nuclei in the skin.

\section{5-ALA/DPPC-induced PpIX accumulation in ex vivo mice skin model}

We further performed confocal microscopy to assay the 5-ALA-induced PpIX accumulation after PDT in the mice skin. The accumulation of PpIX emits a characteristic red fluorescence when exposed to light of the appropriate wavelength. Figure 3 shows confocal images obtained from mechanical skin sections of control- (PBS-), 5-ALA- and 5-ALA/DPPC (0.5\% DPPC)-treated mice. After incubation for 4 hours, the red fluorescence intensity from PpIX of 5-ALA/DPPC-treated skin was obviously stronger than that of 5-ALA. This result indicates that the skin penetration ability of 5-ALA/DPPC was enhanced as compared to the 5-ALA treatment.

\section{5-ALA/DPPC in the mouse xenograft tumor model}

The in vivo mouse xenograft tumor model was established by subcutaneous implantation of B16F10 cells in nude mice. The results showed that the 5-ALA and 5-ALA/DPPC-treated group both had smaller tumor volume than the control group but those differences were not statistically significant within 3 days (Figure 4A). We further analyzed PpIX accumulation in both tumor and healthy skin tissues. Figure 4B shows that PpIX contents in tumor tissues were higher in both 5-ALA and 5-ALA/DPPC $(0.5 \%$ DPPC) groups but not in healthy skin tissues. Furthermore, the 5-ALA/DPPC group had a higher PpIX content in tumor tissue as compared to the 5-ALA group (Figure 4B). This indicates that 5-ALA/DPPC has better skin penetration ability than 5-ALA alone, and this allows for PpIX accumulation in tumor tissue in vivo but we need more mice to assay the influence of 5-ALA/DPPC with respect to tumor volume.
A

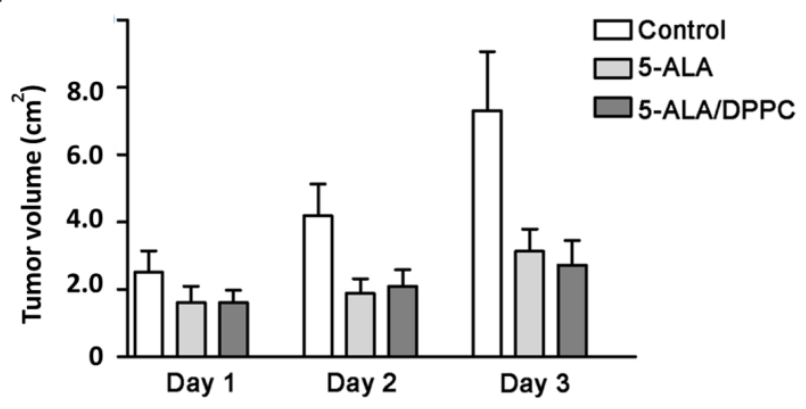

B



Figure 4. 5-ALA/DPPC in the mouse xenograft tumor model. The in vivo mouse xenograft tumor model was established by subcutaneous implantation of B16F10 cells in nude mice. (A) The tumor volume of the 5-ALA and the 5-ALA/DPPC (0.5\% DPPC)-treated groups both had smaller tumor volume than the control group yet didn't show statistically significance from Day 1 to Day 3. (B) PpIX accumulation in tumor and healthy skin tissues shows that the PpIX contents in tumor tissues were higher in both the 5-ALA and 5 -ALA/DPPC groups but not in healthy skin tissues. Furthermore, the 5-ALA/DPPC group had a higher PpIX content in tumor tissue as compared to the 5-ALA only group.

\section{Discussion}

5-ALA is a drug currently used for PDT and has been approved by the U.S. Food and Drug Administration. 5-ALA is a hydrophilic molecule at its physiological $\mathrm{pH}$, and this limits its capacity to cross the stratum corneum of skin. This is concerning 
because skin penetration is a key factor in the efficacy of topical 5-ALA-medicated PDT [5, 15]. Numerous strategies have been proposed to improve penetration, and strategies such as $l$-menthol and its derivative are effective to enhance 5-ALA skin permeation [23]. Yet this problem is still ongoing. Therefore, improving the delivery efficacy for 5-ALA penetration is an urgent matter in 5-ALA-mediated PDT.

As mentioned before, the efficiency of ALA-liposome distribution to tumors is limited by several factors including the physicochemical property of the drugs and the relatively poor perfusion of skin. The lipid components of DPPC liposomes are predominantly DPPC and cholesterol. Based on their biphasic property, DPPC liposomes can act as carriers for hydrophilic drugs such as 5-ALA. Due to the hydrophilic properties of 5-ALA, it can be entrapped in the aqueous phase of liposomes and this brings about the limitation of the entrapment efficiency [18]. Therefore, DPPC was substituted as components of liposomes to increase the entrapment efficiency. In this study, we used DPPC liposomes as vehicles and tested their delivery efficacy of 5-ALA-medicated PDT both in vitro and in vivo. We found that the 5-ALA/DPPC formulation reduced cell viability, mitochondria membrane potential, and enhanced intracellular ROS accumulation as compared to 5-ALA alone in vitro. In addition, the 5-ALA/DPPC formulation also showed better skin penetration ability as compared with 5-ALA in our ex vivo data. In the mouse xenograft tumor models, 5-ALA/DPPC enhanced PpIX accumulation in tumor tissues only and not in healthy skin. Although it reduced tumor volume, it did not do so to a statistical significant extent as compared to 5-ALA. This may be due to the xenograft tumor cell number, tumor depth, or the day of measurement; the exact reason for this still requires further investigations.

The skin distribution of topically applied 5-ALA depends on many parameters, such as drug permeability through the stratum corneum, as well as diffusion through the epidermis and dermis (which are related through compound structures), formulation, release from the vehicle, local drug clearance, and the conversion rate of 5-ALA into PpIX [24]. Methods for improvement of 5-ALA delivery in PDT are based on three concepts: (a) formulation changes to enhance penetration or chelate iron; $(b)$ pretreatment with physical penetration enhancers via ultrasound, laser or iontophoresis; and (c) chemical modification of 5-ALA to increase uptake or selective cleavage [5]. Liposomes are one of the best drug delivery systems for low-molecular-weight drugs, imaging agents, peptides, proteins, and nucleic acids
[16]. Liposomes are microscopic vesicles consisting of one or more membrane-like phosphoid bilayers surrounding an aqueous medium with a particle size from 20 to $500 \mathrm{~nm}$.[17] Pierre et al [18] worked on a delivery system for 5-ALA based on liposomes with a lipid composition of cremide (50\%), cholesterol (28\%), palmitic acid $(17 \%)$, and colesteryl sulfate $(5 \%)$. These SCLL encapsulation rate of 5-ALA was $5.7 \%$ with a $400 \mathrm{~nm}$ particle size. Another report showed that the PDT efficacy and the accumulation of 5-ALA-induced PpIX in murine thymic lymphoma cells increased with a decrease in the average diameter of DPPC liposomes.[25] In the present report, we have shown that the entrapment efficiency of 5-ALA/DPPC was about $15 \sim 16 \%$ with a particle size of around 84 to 89 $\mathrm{nm}$, suggesting that 5-ALA encapsulated in DPPC liposomes has a better encapsulation rate and are smaller in size than those encapsulated in SCLLs.

In conclusion, 5-ALA-based PDT in the treatment for cancer has attracted much attention, and improving the delivery efficacy for 5-ALA penetration through the skin is urgent in 5-ALA-mediated PDT as its penetration is quite poor without any aid. In the present study, we found DPPC liposomes are good carriers for 5-ALA delivery and may prove useful in 5-ALA-mediated PDT in the future.

\section{Acknowledgements}

This work is supported by the Ministry of Science and Technology of Taiwan (MOST 104-2314B-037-003, MOST104-2314-B-006-008, MOST104-2119M-038-001, MOST 104-2923-M-006-002-MY3), Spark Taiwan-ANCHOR UNIVERSITY MOST 104-2321-B006-034, Kaohsiung Medical University "Aim for the Top Universities Grant [KMU-TP104G00], [KMU-TP104G01] \& [KMU-TP104G03], and China Medical University (CMU103-N-03), and NSYSU-KMU Joint Research Project (KMUNSYSU105-I001).

\section{Competing Interests}

The authors have declared that no competing interest exists.

\section{References}

1. Geller AC, Annas GD. Epidemiology of melanoma and nonmelanoma skin cancer. Semin Oncol Nurs. 2003; 19: 2-11.

2. Simoes MC, Sousa JJ, Pais AA. Skin cancer and new treatment perspectives: a review. Cancer Lett. 2015; 357: 8-42.

3. Markovic SN, Erickson LA, Rao RD, Weenig RH, Pockaj BA, Bardia A, et al. Malignant melanoma in the 21st century, part 1: epidemiology, risk factors, screening, prevention, and diagnosis. Mayo Clin Proc. 2007; 82: 364-80.

4. Stadler S, Weina K, Gebhardt C, Utikal J. New therapeutic options for advanced non-resectable malignant melanoma. Adv Med Sci. 2015; 60: 83-8.

5. Lopez RF, Lange N, Guy R, Bentley MV. Photodynamic therapy of skin cancer: controlled drug delivery of 5-ALA and its esters. Adv Drug Deliv Rev. 2004; 56: 77-94.

6. Chen HM, Liu CM, Yang H, Chou HY, Chiang CP, Kuo MY. 5-aminolevulinic acid induce apoptosis via NF-kappaB/JNK pathway in human oral cancer Ca9-22 cells. J Oral Pathol Med. 2011; 40: 483-9. 
7. Gao Y, Zhang XC, Wang WS, Yang Y, Wang HL, Lu YG, et al. Efficacy and safety of topical ALA-PDT in the treatment of EMPD. Photodiagnosis Photodyn Ther. 2015; 12: 92-7.

8. Savoia P, Deboli T, Previgliano A, Broganelli P. Usefulness of Photodynamic Therapy as a Possible Therapeutic Alternative in the Treatment of Basal Cell Carcinoma. International journal of molecular sciences. 2015; 16: 23300-17.

9. Dabrowski JM, Arnaut LG. Photodynamic therapy (PDT) of cancer: from local to systemic treatment. Photochemical \& photobiological sciences : Official journal of the European Photochemistry Association and the European Society for Photobiology. 2015; 14: 1765-80.

10. Dijkstra AT, Majoie IM, van Dongen JW, van Weelden $H$, van Vloten WA. Photodynamic therapy with violet light and topical 6-aminolaevulinic acid in the treatment of actinic keratosis, Bowen's disease and basal cell carcinoma. J Eur Acad Dermatol Venereol. 2001; 15: 550-4.

11. Zeitouni NC, Oseroff AR, Shieh S. Photodynamic therapy for nonmelanoma skin cancers. Current review and update. Mol Immunol. 2003; 39: 1133-6.

12. Leman JA, Dick DC, Morton CA. Topical 5-ALA photodynamic therapy for the treatment of cutaneous T-cell lymphoma. Clin Exp Dermatol. 2002; 27: 516-8.

13. Fritsch C, Lehmann P, Stahl W, Schulte KW, Blohm E, Lang K, et al. Optimum porphyrin accumulation in epithelial skin tumours and psoriatic lesions after topical application of delta-aminolaevulinic acid. Br J Cancer. 1999; 79: 1603-8.

14. Wiegell SR, Stender IM, Na R, Wulf HC. Pain associated with photodynamic therapy using 5-aminolevulinic acid or 5-aminolevulinic acid methylester on tape-stripped normal skin. Arch Dermatol. 2003; 139: 1173-7.

15. Zhang LW, Fang YP, Fang JY. Enhancement techniques for improving 5-aminolevulinic acid delivery through the skin. Dermatologica Sinica. 2011; 29: 1-7.

16. Zucker D, Marcus D, Barenholz Y, Goldblum A. Liposome drugs' loading efficiency: a working model based on loading conditions and drug's physicochemical properties. J Control Release. 2009; 139: 73-80.

17. Fang JY. Nano- or submicron-sized liposomes as carriers for drug delivery. Chang Gung Med J. 2006; 29: 358-62.

18. Pierre MB, Tedesco AC, Marchetti JM, Bentley MV. Stratum corneum lipids liposomes for the topical delivery of 5-aminolevulinic acid in photodynamic therapy of skin cancer: preparation and in vitro permeation study. BMC Dermatol. 2001; $1: 5$.

19. Sparsa A, Bellaton $\mathrm{S}$, Naves $\mathrm{T}$, Jauberteau MO, Bonnetblanc JM, Sol V, et al. Photodynamic treatment induces cell death by apoptosis or autophagy depending on the melanin content in two B16 melanoma cell lines. Oncol Rep. 2013; 29: 1196-200.

20. Chen J, Cheng D, Li J, Wang Y, Guo JX, Chen ZP, et al. Influence of lipid composition on the phase transition temperature of liposomes composed of both DPPC and HSPC. Drug development and industrial pharmacy. 2013; 39: 197-204.

21. Ickowicz Schwartz D, Gozlan Y, Greenbaum L, Babushkina T, Katcoff DJ, Malik Z. Differentiation-dependent photodynamic therapy regulated by porphobilinogen deaminase in B16 melanoma. Br J Cancer. 2004; 90: 1833-41.

22. Mroz P, Yaroslavsky A, Kharkwal GB, Hamblin MR. Cell death pathways in photodynamic therapy of cancer. Cancers (Basel). 2011; 3: 2516-39.

23. Tokuoka Y, Suzuki M, Ohsawa Y, Ochiai A, Ishizuka M, Kawashima N. Enhancement in skin permeation of 5-aminolevulinic acid using 1-menthol and its derivatives. Drug development and industrial pharmacy. 2008; 34: 595-601.

24. Svaasand LO, Wyss P, Wyss MT, Tadir Y, Tromberg BJ, Berns MW. Dosimetry model for photodynamic therapy with topically administered photosensitizers. Lasers Surg Med. 1996; 18: 139-49.

25. Kosobe T, Moriyama E, Tokuoka Y, Kawashima N. Size and surface charge effect of 5-aminolevulinic acid-containing liposomes on photodynamic therapy for cultivated cancer cells. Drug development and industrial pharmacy. 2005; 31: 623-9. 